\title{
Surgical Outcomes of High-Grade Spinal Cord Gliomas
}

\author{
Toshitaka Seki ${ }^{1}$, Kazutoshi Hida ${ }^{2}$, Syunsuke Yano ${ }^{2}$, Takeshi Aoyama ${ }^{3}$, Izumi Koyanagi ${ }^{4}$, Kiyohiro Houkin ${ }^{1}$ \\ ${ }^{1}$ Department of Neurosurgery, Hokkaido University Graduate School of Medicine, Sapporo, Hokkaido, Japan \\ ${ }^{2}$ Sapporo Azabu Neurosurgical Hospital, Sapporo, Hokkaido, Japan \\ ${ }^{3}$ Department of Orthopedic Surgery, Teine Keijinkai Hospital, Sapporo, Hokkaido, Japan \\ ${ }^{4}$ Hokkaido Neurosurgical Memorial Hospital, Sapporo, Hokkaido, Japan
}

Study Design: A retrospective study.

Purpose: The purpose of this study was to obtain useful information for establishing the guidelines for treating high-grade spinal cord gliomas.

Overview of Literature: The optimal management of high-grade spinal cord gliomas remains controversial. We report the outcomes of the surgical management of 14 high-grade spinal glioma.

Methods: We analyzed the outcomes of 14 patients with high-grade spinal cord gliomas who were surgically treated between 1989 and 2012. Survival was charted with the Kaplan-Meier plots and comparisons were made with the log-rank test.

Results: None of the patients with high-grade spinal cord gliomas underwent total resection. Subtotal resection was performed in two patients, partial resection was performed in nine patients, and open biopsy was performed in three patients. All patients underwent postoperative radiotherapy and six patients further underwent radiation cordotomy. The median survival time for patients with high-grade spinal cord gliomas was 15 months, with a 5 -year survival rate of $22.2 \%$. The median survival time for patients with World Health Organization grade III tumors was 25.5 months, whereas the median survival time for patients with glioblastoma multiforme was 12.5 months. Both univariate and multivariate Cox proportional hazards models demonstrated a significant effect only in the group that did not include cervical cord lesion as a factor associated with survival ( $p=0.04$ and 0.03 ).

Conclusions: The surgical outcome of patients diagnosed with high-grade spinal cord gliomas remains poor. Notably, only the model which excluded cervical cord lesions as a factor significantly predicted survival.

Keywords: Glioma; Intramedullary tumor; Outcome; Radiation therapy; Spinal cord

\section{Introduction}

Intramedullary spinal cord tumors are rare entities, accounting for $2 \%$ to $10 \%$ of all central nervous system tumors [1-3] and for $15 \%$ of primary intradural spinal tumors in adults. Seventy percent of these tumors are tumors of low malignant potential, such as low-grade astrocytomas and ependymomas [2,4]. In a recent study of primary spinal cord tumors diagnosed between 1998 and 2002, 69\% were non-malignant and 31\% were malignant [5]. This supports the notion that malignant spinal cord tumors are relatively rare. The incidence of spinal cord

\footnotetext{
Received Mar 11, 2015; Accepted Mar 13, 2015

Corresponding author: Toshitaka Seki

Department of Neurosurgery, Hokkaido University Graduate School of Medicine,

North-15 West-7, Kita-ku, Sapporo 060-8638, Hokkaido, Japan

Tel: +81-11-706-5987, Fax: +81-11-708-7737, E-mail: toseki1@hotmail.com
} 
Table 1. Characteristics and outcomes of 14 patients with high-grade spinal cord gliomas

\begin{tabular}{|c|c|c|c|c|c|c|c|c|c|c|c|}
\hline No. & $\begin{array}{l}\text { WHO } \\
\text { grade }\end{array}$ & Age/sex & $\begin{array}{l}\text { Duration of } \\
\text { symptom } \\
\text { (mo) }\end{array}$ & $\begin{array}{c}\text { Preoperative } \\
\text { MMS }\end{array}$ & Localization & Resection & XRT & $\mathrm{RC}$ & Chemo. & Outcome & $\begin{array}{l}\text { Last } \\
\text { follow-up } \\
\text { (mo) }\end{array}$ \\
\hline 1 & III & 53/male & 84 & 4 & T8-11 & Biopsy & Y & $\mathrm{N}$ & Y & $\mathrm{N} / \mathrm{A}$ & 85 \\
\hline 2 & III & 12/female & 0.4 & 2 & T7-11 & Partial & Y & Y & $\mathrm{N}$ & $D$ & 14 \\
\hline 3 & III & 18/female & 1 & 2 & C1-4 & Subtotal & $Y$ & $\mathrm{~N}$ & Y & $D$ & 7 \\
\hline 4 & III & 54/male & 60 & 2 & T8-12 & Partial & Y & $\mathrm{N}$ & Y & D & 60 \\
\hline 5 & III & $34 /$ male & 3 & 2 & C4-6 & Partial & Y & $\mathrm{N}$ & $\mathrm{N}$ & D & 36 \\
\hline 6 & III & $31 /$ female & 14 & 2 & T12-conus & Partial & Y & Y & Y & A & 149 \\
\hline 7 & III & 53/male & 4 & 2 & T10-conus & Partial & Y & Y & $N$ & $\mathrm{~N} / \mathrm{A}$ & 53 \\
\hline 8 & III & $66 /$ male & 6 & 4 & T7-conus & Biopsy & Y & Y & Y & $A$ & 2 \\
\hline 9 & III & 15/male & 2 & 4 & $\mathrm{C} 4-\mathrm{T} 2$ & Partial & Y & $\mathrm{N}$ & $\mathrm{N}$ & D & 10 \\
\hline 10 & III & $75 /$ male & 2 & 2 & C4-7 & Biopsy & Y & $\mathrm{N}$ & Y & D & 15 \\
\hline 11 & IV & 13/male & 24 & 4 & C3-T1 & Partial & Y & $\mathrm{N}$ & $\mathrm{N}$ & $\mathrm{N} / \mathrm{A}$ & 2 \\
\hline 12 & IV & 23/female & 4 & 4 & T9-conus & Subtotal & Y & Y & $\mathrm{N}$ & D & 48 \\
\hline 13 & IV & $37 /$ male & 1.5 & 3 & T2-5 & Partial & Y & Y & Y & D & 15 \\
\hline 14 & IV & 55/male & 3 & 5 & T3-11 & Partial & Y & $N$ & Y & D & 10 \\
\hline
\end{tabular}

WHO, World Health Organization; MMS, modified McCormick score; XRT, radiotherapy; RC, radiation cordotomy; Chemo., chemotherapy; T, thoracic; N/A, not available; $D$, dead; C, cervical; $A$, alive.

gliomas has been reported to be approximately 0.22 per 100,000 [5]. The most common gliomas of the spinal cord are astrocytomas and ependymomas, with ependymomas accounting for 60 to $80 \%$ of all gliomas [6,7]. Given the rarity of primary malignant spinal cord gliomas, the optimal treatment guidelines have yet to be established. The aim of this study was to obtain useful information for establishing the guidelines for decision-making in the treatment of high-grade spinal cord gliomas by examining the clinical records of patients treated at Hokkaido University Hospital.

\section{Materials and Methods}

Clinical outcomes of 14 patients who underwent surgery for high-grade spinal cord gliomas at Hokkaido University Hospital between January 1989 and December 2012 were assessed (Table 1). Of these, 10 were males and 4 were females, and their ages ranged from 12 to 75 years at the time of surgery (mean age, 38.5 years). The follow-up period ranged from 2 to 149 months (mean, 36.1 months). Ten patients had World Health Organization (WHO) grade III tumors, including eight patients with anaplastic astrocytomas, one patient with anaplastic oligoastro- cytoma, and one patient with anaplastic ependymoma. Four patients had WHO grade IV tumors. Tumors were located at the cervical level in three patients, at the cervicothoracic level in two patients, at the thoracic level in six patients, and at the thoracolumbar level in three patients. The extent of resection was defined as macroscopic gross total resection $(100 \%)$, subtotal resection $(>90 \%)$, partial resection $(<90 \%)$, or open biopsy. Following laminoplasty in the cases with involvement of the cervical region or laminectomy in the cases with involvement of both thoracic and thoraco-lumbar regions, tumors were surgically removed via a posterior median approach by subtotal resection in two patients, partial resection in nine patients, and open biopsy in three patients. Furthermore, we used an ultrasonic surgical device (SonopeT UST-2001, Miwa Tec, Kawasaki, Japan) to reduce the internal volume, and performed intraoperative monitoring of motor evoked potentials in all patients. After removal of the tumor, we performed duraplasty with a Gore-Tex (W. L. Gore \& Associates Inc., Flagstaff, AZ, USA) membrane. None of the patients underwent gross total resection. For postoperative adjuvant therapy, conventional radiation therapy (4050 Gy in 20-25 fractions) was performed in all patients. Patients who did not have cervical cord lesions were 
candidates for a radiotherapy boost for dose escalation. Eight patients underwent chemotherapy (WHO grade III tumors, 6 patients; WHO grade IV tumors, 2 patients).

Survival was set as the period from initial surgery to the date of death or last follow-up. The length of survival from time of surgery was charted with the Kaplan-Meier method [8] and compared via univariate and multivariate analyses. Hazard ratios were determined by proportional hazards analysis (Cox model) for continuous covariates and stratified variables. Probability values $<0.05$ were considered statistically significant. All data were analyzed using commercially available software (StatView, SAS Institute Inc., Cary, NC, USA).

\section{Results}

Among the 14 patients with high-grade spinal cord gliomas, only two patients were alive at the time of initiation of this study (Table 1). In the present study, only one patient showed dissemination in the brain. Furthermore, there was no recurrence of the tumor in one patient who had anaplastic astrocytoma. One patient had died of other cancer. The remaining patients showed regrowth of the tumor. In none of the patients, the tumor had disseminated to a different location in the spinal cord. The survival rate for the 14 patients was $22.2 \%$ and the median survival time was 15.0 months (range, 2-149 months) (Fig. 1). Preoperative median modified McCormick score $[9,10]$ was 2.5. Seven patients presented with grade II neurological status, one patient presented with grade III neurological status, five patients presented with grade IV neurological status, and one patient presented with grade $\mathrm{V}$ neurological status. According to the postoperative modified McCormick score, three patients presented with grade II neurological status, two patients presented with grade III neurological status, seven patients presented with grade IV neurological status, and one patient presented with grade V neurological status. Postoperative median modified McCormick score was 4 . The median survival time for patients with WHO grade III tumors was 25.5 months (range, 2-149 months), while the median survival time for patients with WHO grade IV tumors was 12.5 months (range, 2-48 months); however, there was no significant difference between the two groups (log-rank test, $p=0.33$ ) (Fig. 2). After excluding patients with cervical spinal cord gliomas, the median survival time was 48 months, whereas it was significantly shorter at ten months after including all patients with spinal cord gliomas ( $p=0.04$ ) (Fig. 3).

All patients with high-grade spinal cord gliomas received postoperative conventional radiation therapy. Furthermore, six patients (43\%) underwent both conventional radiation therapy and postoperative radiation cordotomy (WHO grade III tumors, 4 patients; WHO grade IV tumors, 2 patients). However, patients with cervical spinal cord lesions did not undergo radiation, high-dose cordotomy. Patients who underwent surgery, conventional radiation therapy, radiation, and high-dose cordotomy had a mean survival time of 31.5 months,

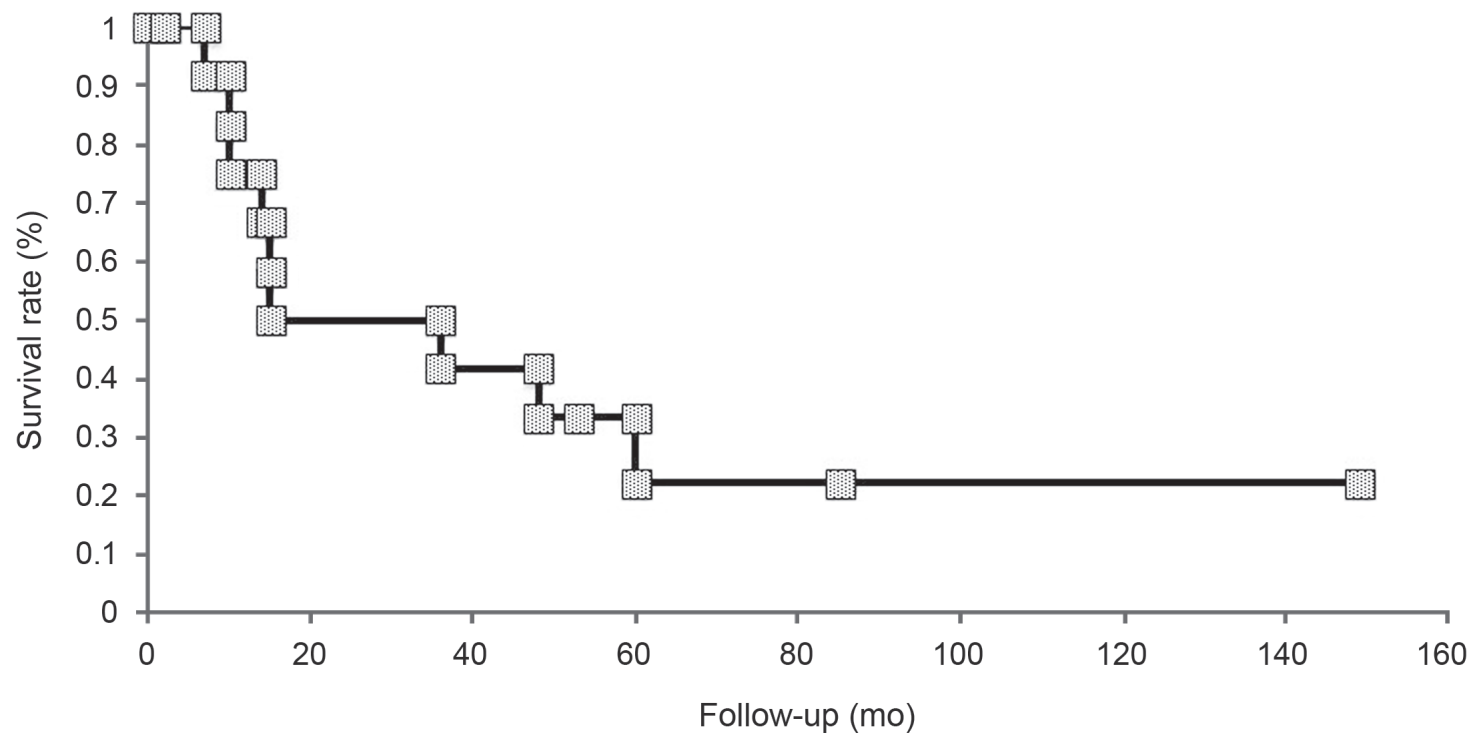

Fig. 1. The survival rate of patients with high-grade spinal cord gliomas. 


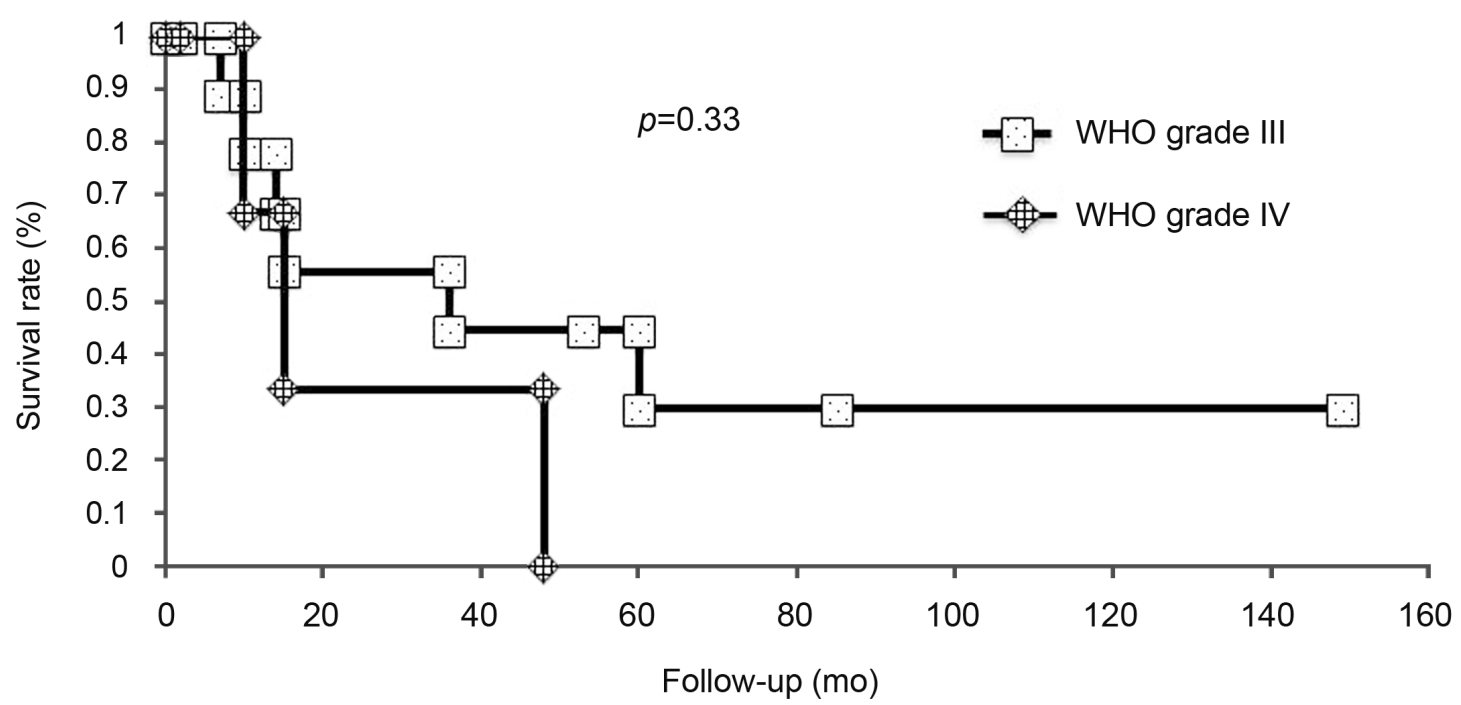

Fig. 2. The survival rate of patients with (World Health Organization, WHO) grade III and grade IV tumors. The survival rate of patients with WHO grade III tumors was higher than that of patients with WHO grade IV tumors; however, the difference was not significant $(p=0.33)$.

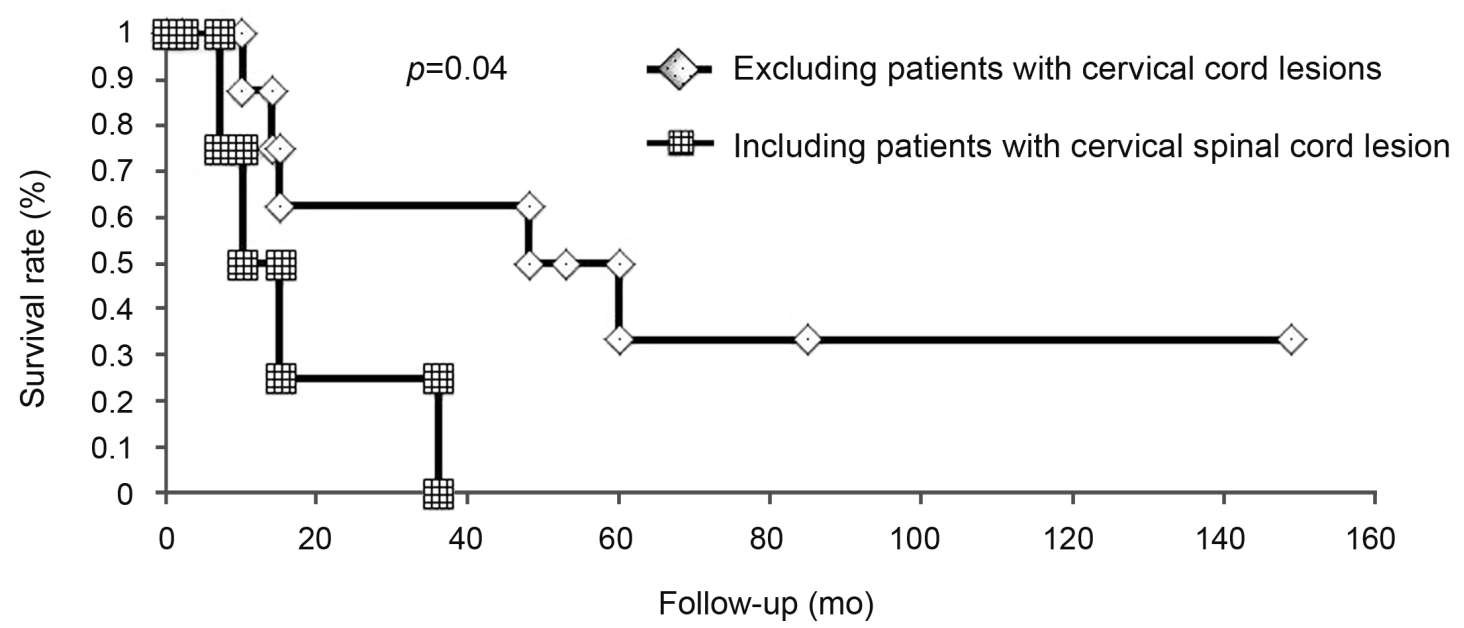

Fig. 3. Impact of the pathologic grade on survival. There was a significant difference in survival after excluding patients with cervical cord lesions compared to that after including patients with cervical cord lesions $(p=0.04)$.

whereas those who underwent surgery and conventional radiation therapy alone had a shorter mean survival time of 12.5 months; however, the difference was not significant $(p=0.38)$ (Fig. 4). Six patients with WHO grade III tumors and two patients with WHO grade IV tumors received postoperative adjuvant chemotherapy. Among the patients with WHO grade III tumors, three patients were managed with 1-(4-amino-2-methyl-5-pyrimidinyl) methyl-3-(2-chloroethyl)-3-nitrosourea (ACNU) and the remaining three patients were treated with temozolomide. On the other hand, two patients with WHO grade IV tumors were administered ACNU. None of the patients with high-grade spinal cord gliomas were treated with surgery alone. When WHO grading and tumor location were included in the Cox model, the model which excluded patients with cervical cord lesions significantly predicted survival $(p=0.03$ ) (Table 2 ).

\section{Illustrative case}

A 31-year-old female developed numbness in her left lower limb and underwent a preoperative neurological assessment. The assessment revealed left leg paresis, sensory disturbance in the left leg, a neurogenic bladder, and rec- 


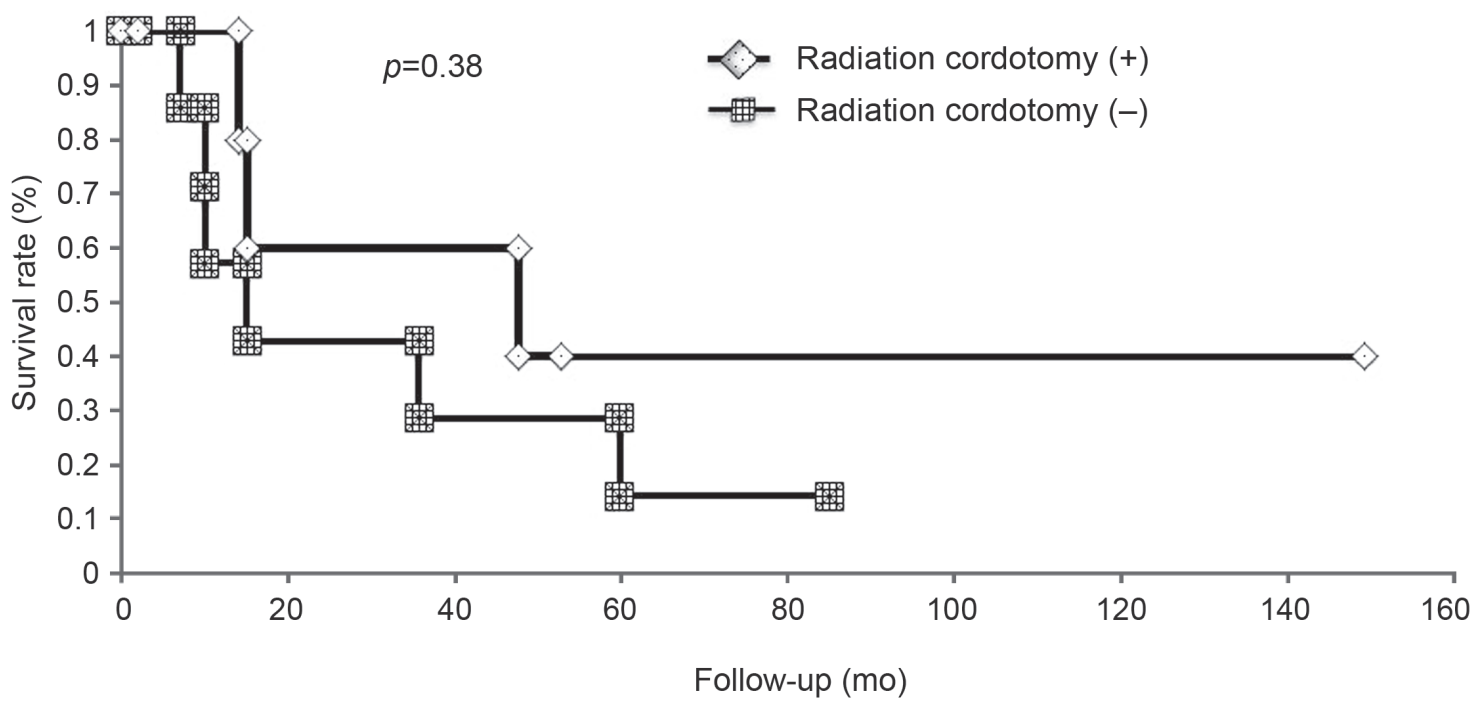

Fig. 4. Effect of radiation cordotomy on patient survival. There was no significant difference in survival of patients who underwent radiation cordotomy or non-radiation cordotomy.

Table 2. Prognostic factors with high-grade spinal cord gliomas

\begin{tabular}{lccc} 
& \multicolumn{3}{c}{ Hazard } \\
\cline { 2 - 4 } Variable & $p$-value & Ratio & $95 \%$ Confidence interval \\
\cline { 2 - 4 } Histology $^{\text {al }}$ & 0.08 & 0.13 & $0.013-1.263$ \\
Tumor localization $^{\text {b) }}$ & $0.03^{\text {cl }}$ & 0.78 & $0.008-0.807$ \\
\hline
\end{tabular}

${ }^{a}$ World Health Organization grade III vs. IV; ${ }^{b /}$ Including the cervical spinal cord vs. not including the cervical spinal cord; ${ }^{\text {c)} C a l c u l a t e d ~ u s i n g ~ C o x ~ p r o p o r-~}$ tional hazard analysis.
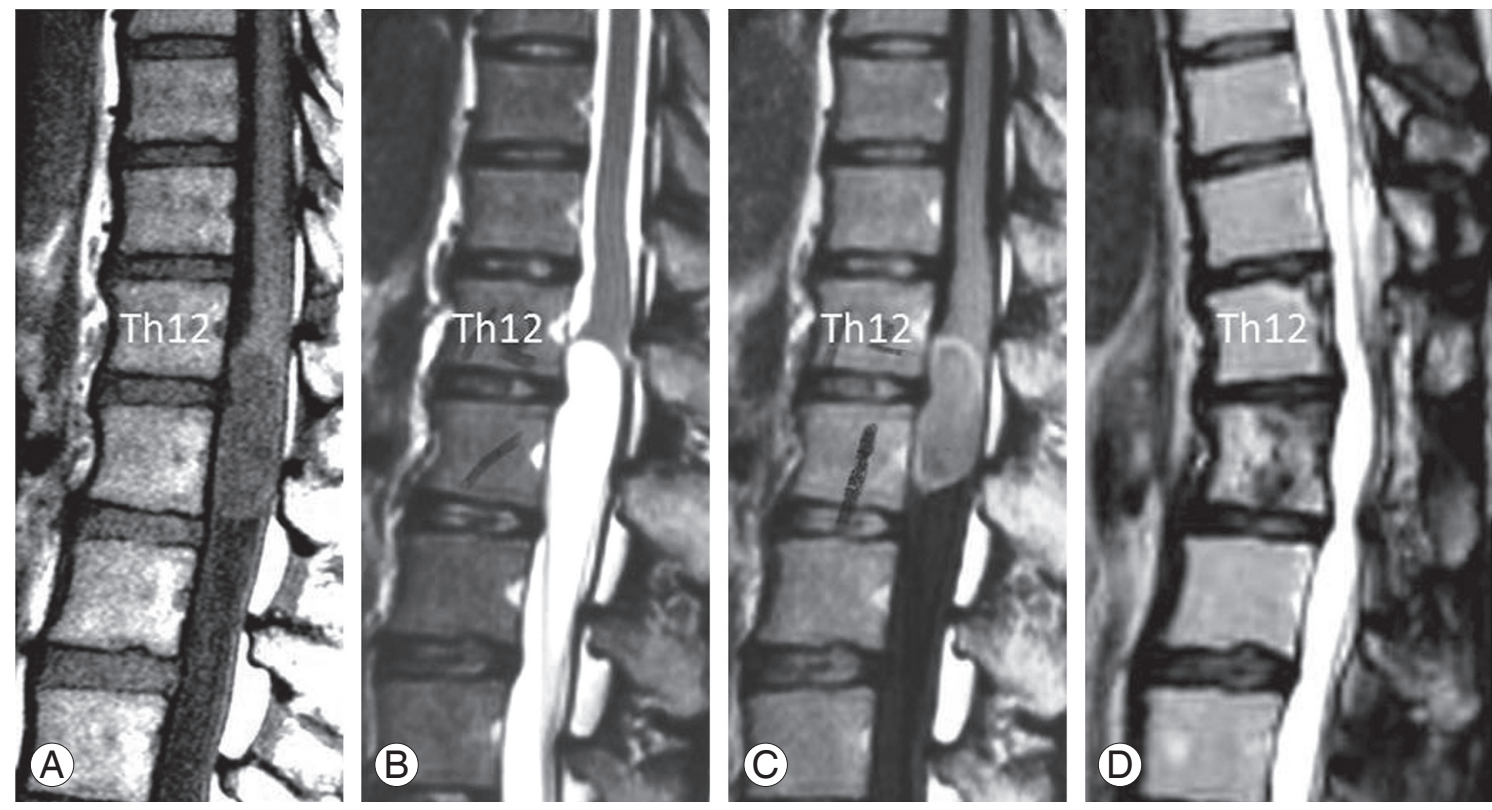

Fig. 5. Anaplastic astrocytoma in a 31-year-old female. (A-C) Preoperative and (D) postoperative magnetic resonance findings. (A) T1-weighted, (B, D) T2-weighted, and (C) gadolinium (D) T2-weighted image at the final follow-up period. 
tal disturbance. Preoperative T1- and T2-weighted magnetic resonance (MR) images revealed diffuse swelling of the spinal cord with iso- and high-signal intensities at the conus medullaris (Fig. 5A, B), and gadolinium-enhanced T1-weighted MR images revealed ring-like enhancement (Fig. 5C). Anaplastic astrocytoma was identified and the patient underwent partial resection. For adjuvant therapy, the patient received conventional radiotherapy (14 Gy/7 fr) and radiation high-dose cordotomy (60 Gy/10 fr). At 149 months postoperatively, there were no signs of tumor recurrence on MR images (Fig. 5D). Despite being wheelchair-bound and having severe spinal cord atrophy, the patient has returned to her original place of employment.

\section{Discussion}

High-grade spinal cord gliomas are extremely rare, and there is no standard treatment to date. The prognosis for patients with these tumors who undergo surgery and adjuvant radiation therapy and/or chemotherapy is reportedly poor [11-14]. In the present study, we compared the survival time, according to the pathologic grade in patients with WHO grade III and WHO grade IV tumors (Fig. 2). Although there was no significant difference in survival between patients with two grades of tumors, patients with anaplastic gliomas fared better, with a median survival time of 25.5 months compared with 12.5 months for patients with glioblastoma multiforme (log-rank test, $p=0.33$ ).

We also compared survival by either including or excluding patients with cervical cord lesions, without taking the pathologic grade into account (Fig. 3). After excluding patients with cervical cord lesions, survival of patients with high-grade spinal cord gliomas was 48 months, whereas it was ten months after including all patients with spinal cord high-grade gliomas (log-rank test, $p=0.04$ ). Similarly, Nakamura et al. [14] reported that patients with cervical cord tumors had a significantly worse prognosis than those with thoracic cord tumors. One possible reason for this is that it takes a thoracic tumor longer to reach the upper cervical cord; thus causing central respiratory failure compared to a cervical cord tumor.

There is no consensus regarding the most appropriate surgical intervention for treating high-grade spinal cord gliomas. While some surgeons endorse aggressive tumor resection [14-17], others have reported that biopsy alone yields better outcomes $[12,13,18]$. McGirt et al. [17] re- ported that radical resection was associated with a trend of prolonged survival for patients with anaplastic spinal cord gliomas but not for those with glioblastoma multiforme. Patients with anaplastic spinal cord gliomas who underwent radical resection had increased overall survival rates compared with those who underwent subtotal resection [17]. In the present study, partial resection (64.3\%) or open biopsy (21.4\%) was performed in most patients. If resection rates had been higher, longer survival periods may have been obtained. On the other hand, Minehan et al. [13] reported that the extent of tumor resection did not impact patient survival, and in fact, there was a trend for poorer survival in patients who underwent more aggressive resection compared to those who underwent biopsy alone. However, biopsy or partial resection to avoid neurological deterioration may not be appropriate even for patients with high-grade spinal cord gliomas, as both functional outcomes and survival rates were poor after these procedures. Thus, to improve the survival rate, we suggest radical resection, particularly if the tumor is located in the thoracic cord, although it is quite difficult to distinguish such tumors from the normal spinal cord.

The efficacy of adjuvant radiotherapy for the treatment of high-grade spinal cord gliomas has yet to be determined [13,19]. Minehan et al. [13] reported that postoperative radiotherapy significantly enhanced overall survival in patients with high-grade spinal cord gliomas, although lower limb and bladder function needed to be sacrificed. Katoh et al. [20] reported that the 5-year survival rate for patients with high-grade gliomas who received hypofractionated radiotherapy was $67 \%$, and it was $35 \%$ for those who received conventional radiation therapy. In the present study, there was no significant difference in the survival rate between patients who received adjuvant radiotherapy and those who did not receive adjuvant radiotherapy, regardless of the pathological grade (data not shown). Similarly, there were no significant differences between patients who received radiation cordotomy and those who did not receive radiation cordotomy (log-rank test, $p=0.38$ ). However, patients who underwent surgery and radiation cordotomy survived longer than those who underwent surgery and conventional radiation therapy alone (31.5 months vs. 12.5 months). This suggests that in the treatment of high-grade spinal cord gliomas, particularly in cases of thoracic cord tumors, the combination of radical resection and radiation cordotomy may prolong survival. 


\section{Conclusions}

Total surgical resection of high-grade gliomas is rarely possible given the infiltrative behavior of this tumor type. Tumor location was significantly correlated with the survival rate. Notably, radiation cordotomy for tumors located below the level of the cervical cord moderately impacted survival. High-grade spinal cord gliomas are rare, and thus, the number of patients who could be assessed at a single facility is limited. Thus, a prospective multicenter study with a unified treatment strategy is warranted in the future.

\section{Conflict of Interest}

No potential conflict of interest relevant to this article was reported.

\section{References}

1. Hsu S, Quattrone M, Ostrom Q, Ryken TC, Sloan $\mathrm{AE}$, Barnholtz-Sloan JS. Incidence patterns for primary malignant spinal cord gliomas: a Surveillance, Epidemiology, and End Results study. J Neurosurg Spine 2011;14:742-7.

2. Raco A, Esposito V, Lenzi J, Piccirilli M, Delfini R, Cantore G. Long-term follow-up of intramedullary spinal cord tumors: a series of 202 cases. Neurosurgery 2005;56:972-81.

3. Stein BM, McCormick PC. Intramedullary neoplasms and vascular malformations. Clin Neurosurg 1992;39:361-87.

4. Schwartz TH, McCormick PC. Intramedullary ependymomas: clinical presentation, surgical treatment strategies and prognosis. J Neurooncol 2000;47:2118.

5. Schellinger KA, Propp JM, Villano JL, McCarthy BJ. Descriptive epidemiology of primary spinal cord tumors. J Neurooncol 2008;87:173-9.

6. Henson JW. Spinal cord gliomas. Curr Opin Neurol 2001;14:679-82.

7. Shrivastava RK, Epstein FJ, Perin NI, Post KD, Jallo GI. Intramedullary spinal cord tumors in patients older than 50 years of age: management and outcome analysis. J Neurosurg Spine 2005;2:249-55.
8. Kaplan EL, Meier P. Nonparametric estimation from incomplete observations. J Am Stat Assoc 1958;53: 457-81.

9. Constantini S, Miller DC, Allen JC, Rorke LB, Freed D, Epstein FJ. Radical excision of intramedullary spinal cord tumors: surgical morbidity and longterm follow-up evaluation in 164 children and young adults. J Neurosurg 2000;93:183-93.

10. McCormick PC, Stein BM. Intramedullary tumors in adults. Neurosurg Clin N Am 1990;1:609-30.

11. Adams H, Avendano J, Raza SM, Gokaslan ZL, Jallo GI, Quinones-Hinojosa A. Prognostic factors and survival in primary malignant astrocytomas of the spinal cord: a population-based analysis from 1973 to 2007. Spine (Phila Pa 1976) 2012;37:E727-35.

12. Innocenzi G, Salvati M, Cervoni L, Delfini R, Cantore G. Prognostic factors in intramedullary astrocytomas. Clin Neurol Neurosurg 1997;99:1-5.

13. Minehan KJ, Brown PD, Scheithauer BW, Krauss WE, Wright MP. Prognosis and treatment of spinal cord astrocytoma. Int J Radiat Oncol Biol Phys 2009; 73:727-33

14. Nakamura M, Chiba K, Ishii K, et al. Surgical outcomes of spinal cord astrocytomas. Spinal Cord 2006; 44:740-5.

15. Cooper PR, Epstein F. Radical resection of intramedullary spinal cord tumors in adults: recent experience in 29 patients. J Neurosurg 1985;63:492-9.

16. Epstein FJ, Farmer JP, Freed D. Adult intramedullary astrocytomas of the spinal cord. J Neurosurg 1992;77: 355-9.

17. McGirt MJ, Goldstein IM, Chaichana KL, Tobias ME, Kothbauer KF, Jallo GI. Extent of surgical resection of malignant astrocytomas of the spinal cord: outcome analysis of 35 patients. Neurosurgery 2008;63:55-60.

18. Sandler HM, Papadopoulos SM, Thornton AFJ, Ross DA. Spinal cord astrocytomas: results of therapy. Neurosurgery 1992;30:490-3.

19. Kim MS, Chung CK, Choe G, Kim IH, Kim HJ. Intramedullary spinal cord astrocytoma in adults: postoperative outcome. J Neurooncol 2001;52:85-94.

20. Katoh N, Shirato H, Aoyama H, et al. Hypofractionated radiotherapy boost for dose escalation as a treatment option for high-grade spinal cord astrocytic tumor. J Neurooncol 2006;78:63-9. 